\title{
Visual Perception of Pain in Neonatal Face Images
}

\author{
Lucas Pereira Carlini \\ Departamento de Engenharia Elétrica \\ Centro Universitário FEI \\ São Bernardo do Campo, Brasil \\ lucaspcarlini10@gmail.com
}

\author{
Tatiany M. Heideirich \\ Departamento de Pediatria \\ Universidade Federal de São Paulo \\ São Paulo, Brasil \\ tatianyms@gmail.com
}

\author{
Rita C. X. Balda \\ Departamento de Pediatria \\ Universidade Federal de São Paulo \\ São Paulo, Brasil \\ ritabalda@live.com
}

\author{
Marina C. M. Barros \\ Departamento de Pediatria \\ Universidade Federal de São Paulo \\ São Paulo, Brasil \\ marinamoraesbarros@gmail.com
}

\author{
Ruth Guinsburg \\ Departamento de Pediatria \\ Universidade Federal de São Paulo \\ São Paulo, Brasil \\ ruth.guinsburg@gmail.com
}

\author{
Carlos Eduardo Thomaz \\ Departamento de Engenharia Elétrica \\ Centro Universitário FEI \\ São Bernardo do Campo, Brasil \\ cet@fei.edu.br
}

\begin{abstract}
More than 500 painful intervetions are carried out during the hospitalization of a newborn baby in an intensive medical care unit. In these situations, there is, however, a challenging difficulty to identify pain, owing to the unlikeliness of direct and objective verbal communication commonly used among adults. This work is part of an on-going research that aims to develop a computational framework to interpreting and recognizing patterns on face images for automatic assessment of neonatal procedural pain. We believe that such investigation might provide relevant information to understand the relation between neonatal facial features and procedural pain and, consequently, helping health professionals in the corresponding clinical practice.
\end{abstract}

Index Terms-Newborn Face Images. Eye-tracking. Facial Perception. Pain.

\section{INTRODUÇÃO}

Para seres humanos adultos, indicações objetivas da dor são feitas por comunicação verbal e também por expressões corporais. No entanto, no estágio inicial da vida, o ser humano não possui a capacidade de verbalizar a sua dor. Para o Recém-Nascido (RN), a identificação da presença da dor e a quantificação da mesma é dificultada, levando essa tarefa à análise de ações e reações, não necessariamente objetivas, frente ao estímulo causador da dor.

Neste contexto, diversos trabalhos têm sido desenvolvidos nos últimos anos para automatizar a avaliação da dor do RN [1] [2] [3]. Em particular, a metodologia desenvolvida em [2] mostrou-se bem sucedida no que se refere à possibilidade de automatizar a avaliação da dor do RN por meio da captura de imagens de face, da decodificação das mesmas e da classificação do resultado, como dor presente ou ausente em intervalo de tempo quase instantâneo.

Entretanto, essa metodologia [2] e as demais existentes [1] [3], no estágio em que se encontram, não trazem informações a respeito da relevância das características intrínsecas da face do $\mathrm{RN}$ que definem a dor usadas pelo profissional de saúde para interpretação deste fenômeno, sendo capazes de identificar a dor como presente somente se o recém-nascido expressar-se caracteristicamente por meio de expressões faciais específicas e pré-determinadas.

Visando a prática clínica em questão, a interpretação pelos profissionais de saúde do que o RN expressa é fundamental para que o fenômeno doloroso possa ser identificado, avaliado e se necessário, tratado. Apesar da literatura mostrar que a avaliação da dor difere, por exemplo, entre os profissionais de saúde e entre pais [4] [5], pouco se sabe a respeito da percepção facial humana com relação ao fenômeno dor no RN.

O presente trabalho tem como objetivo estudar pioneiramente as diferentes estratégias oculares empregadas por adultos na avaliação do fenômeno dor presente em neonatos, de maneira que se possua uma compreensão maior a respeito das características intrínsecas da face do RN necessárias para essa avaliação. Especificamente, desenvolve-se aqui um arcabouço computacional para a aquisição dos movimentos oculares humanos em sessões específicas de análise de imagens de faces de $\mathrm{RN}$ a termo (com dor e sem dor) por meio de equipamento de rastreamento de olhar.

\section{CONCEITOS Fundamentais}

\section{A. O Fenômeno da Dor}

O conceito de dor foi primeiramente desenvolvido em 1970 [6]. Esse conceito inclui o critério de ser uma experiência desagradável e subjetiva, com a possibilidade de haver dano físico e estímulo neurológico nociceptivo. Anos mais tarde, o conceito da dor foi ampliado [7] para um conjunto de experiências desagradáveis, podendo ser sensorial ou emocional, associada à lesão real ou potencial dos tecidos, e sendo a mesma totalmente subjetiva.

Com tantos efeitos degradantes à vida das pessoas [8] [9], a dor é tema e motivação de pesquisa de diversos estudiosos e profissionais da saúde [2] [4], e também de outras áreas [1] [3]. Essas pesquisas envolvem a identificação, tratamento e alívio da dor.

Acreditava-se que o RN não tinha a capacidade de sentir dor até quando o mesmo atingisse um ano de vida [10]. 
Dessa maneira, procedimentos em prol do alívio da dor eram raramente realizados em neonatos [10].

No entanto, estudos do final da década de 1980 mostraram que o sistema nervoso fetal está suficientemente maduro para a nocicepção ao redor da $24^{\circ}$ semana de gestação [11]. Além disso, outros estudos também verificaram que as vias inibitórias dos estímulos dolorosos, possuindo a funcionalidade de amenizar a transmissão desses estímulos, ainda não estão completamente desenvolvidas no nascimento, consequentemente, o neonato mostra maior sensibilidade ao estímulo doloroso do que seres humanos adultos [12].

Dessa forma, a identificação da dor em RNs passou a ser feita por meio da análise de ações e reações, de maneira indireta, frente ao estímulo incômodo da dor [1] [2] [3] .

\section{B. Sistema de Codificação Facial Neonatal}

Figura 1: Representação ilustrativa das características que compõem a escala NFCS.

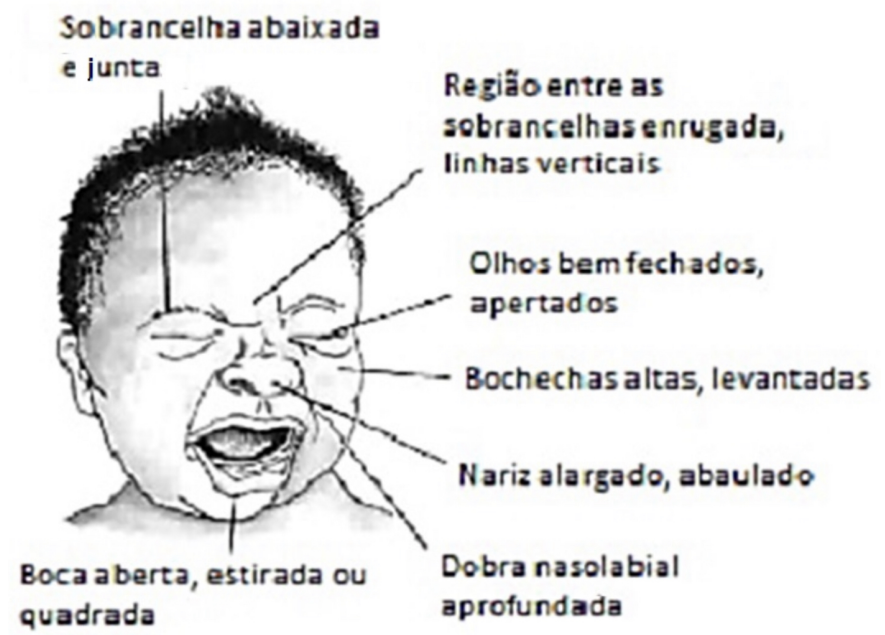

\section{Expressão facial de estresse e dor em um Recém-nascido}

O método mais comum de avaliação de dor em RN utilizado até então na prática clínica se baseia na análise da mímica facial do neonato [13]. A análise da mímica facial fornece informações válidas, sensíveis a respeito da natureza e da intensidade da dor, permitindo uma comunicação eficaz entre o neonato e as pessoas envolvidas em seus cuidados [13].

Dentre as diversas escalas e sistemas de avaliação desse tipo, o mais utilizado, em RNs pré-termo, denomina-se Sistema de Codificação Facial Neonatal (em inglês, Neonatal Facial Coding System - NFCS) [13], que baseia-se na pontuação diante da presença de características específicas na face do neonato [14]. Essas características são mostradas na Figura 1. A presença da dor é verificada quando três ou mais dessas características estão presentes.
As características descritas pelo NFCS somente estão presentes em estímulos dolorosos [14], tornando esse método de quantificação confiável clinicamente [2] [14].

\section{Materiais e Métodos}

\section{A. Participantes}

Os experimentos conduzidos nesse trabalho tiveram a participação de 9 voluntários leigos (não profissionais de saúde), sendo 5 homens e 4 mulheres, com idades entre 17 e 50 anos. Todos saudáveis e capazes para o cumprimento do experimento proposto. Foi obtida concordância de todos os participantes por meio de termo de consentimento livre e esclarecido sobre o estudo.

\section{B. Aquisição do Sinal}

A aquisição do sinal foi feita em uma sala fechada, com iluminação artificialmente controlada dentro das especificações entre 300 e $1000 l x$ e posicionada fora do campo visual do participante. Foi utilizado o equipamento de rastreamento ocular Tobii TX300. O mesmo possui capacidade de captura de dados de $300 \mathrm{~Hz}$ e é composto por um monitor TFT de 23 polegadas com um sistema de iluminação infravermelho e duas câmeras de captura localizadas na parte inferior do equipamento.

Em conjunto com o equipamento de rastreamento ocular, foi utilizado um computador auxiliar com o software de controle Tobii Studio, para a calibração do equipamento e aquisição dos dados. Esse computador auxiliar, de estilo notebook, possui processador Intel Core I7, 16GB de memória RAM, e sistema operacional Microsoft Windows 7.

Figura 2: Posição ideal recomendada.
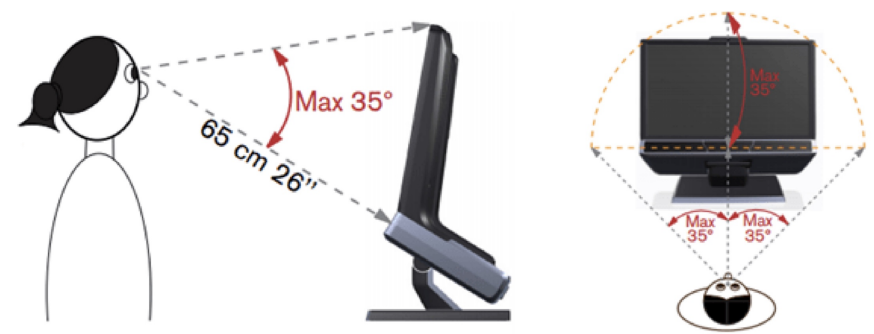

\section{Estímulo Proposto}

Foi criado e desenvolvido um procedimento computacional no qual voluntários realizaram a avaliação da dor em diversas imagens de RNs, por meio de uma escala numérica analógica, em que eram atribuídas notas variando de 0 (RN sem dor) a 10 (RN com dor extrema) para cada imagem de neonato apresentada. Em cada sessão do procedimento, as informações referentes a estratégia ocular de avaliação de cada voluntário eram registradas pelo equipamento de rastreamento de olhar. Houve critério de exclusão relacionado apenas aos diagnósticos de epilepsia (convulsão) e problemas oculares graves. 


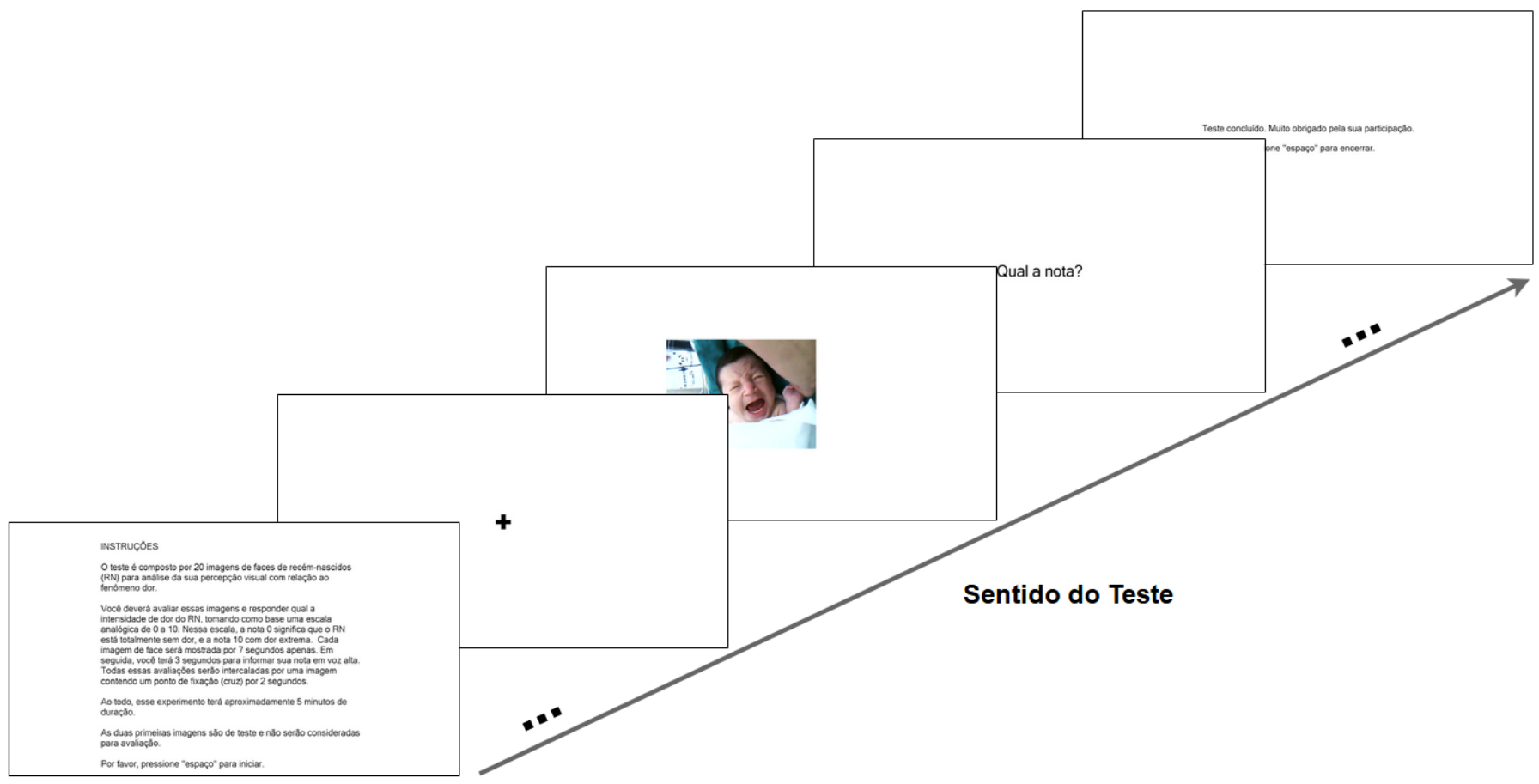

Figura 3: Procedimento computacional de captura do olhar dos voluntários.

Para a implementação do teste proposto, utilizou-se um banco de imagens de faces construído por pesquisadores e profissionais de saúde, não sendo esse banco de livre acesso. A autorização de uso dessas imagens teve consentimento assinado dos familiares ou responsáveis, e o projeto de construção e análise dessas imagens foi aprovado por Comitê de Ética afim (1299/09, 3.116.151 e 3.116.146).

Foram selecionadas 20 imagens referentes a 10 RNs. Cada par de imagem é composto por uma imagem do neonato em repouso e outra imagem após punção em procedimento doloroso.

O procedimento se inicia após a calibração do equipamento Tobii TX300 com o participante, conforme Figura 2. Depois, exibe-se inicialmente uma tela de instruções referentes ao experimento, permitindo que todos os voluntários recebam exatamente as mesmas instruções. Após a tela de instruções, o participante realiza duas avaliações como teste. Os resultados obtidos nas mesmas não são considerados no estudo final, pois apenas possuem como finalidade a familiarização do participante com o procedimento proposto. Em seguida, as avaliações propriamente se iniciam. As imagens de face que são avaliadas pelos participantes são mostradas em sequência aleatória e expostas durante 7 segundos cada. A estrutura e sequência de imagens do experimento são mostradas na Figura 3.

As imagens de face foram intercaladas no experimento por uma tela que contêm apenas uma cruz como ponto de fixação central. O tempo de exibição sugerido para o ponto de fixação é de 2 segundos [15]. Essa intercalação se justifica pelo motivo de que a estratégia ocular de todos os participantes terá início no mesmo ponto da tela, não prejudicando as primeiras sacadas (movimento ocular entre pontos de fixação do olhar) da avaliação. Deve-se destacar, no entanto, que as fixações realizadas pelos participantes nos $300 \mathrm{~ms}$ iniciais do estímulo foram descartados. Esse filtro é justificado por conta da inércia de olhar causada pela cruz de fixação mostrada previamente ao estímulo [15].

Para cada imagem de face, o participante teve 3 segundos para atribuir uma nota referente a imagem exibida. O tempo total para a realização do experimento foi de, aproximadamente, 5 minutos para cada voluntário.

\section{RESUltados}

A Figura 4 mostra a dispersão das notas atribuídas por todos os voluntários após avaliação de cada imagem de recémnascido. Nesta figura, é possível verificar a avaliação condizente da presença do fenômeno dor por parte da maioria dos voluntários, evidenciada pelas notas de maior valor atribuídas às imagens de RNs após punção e pelas notas de valores inferiores às imagens de $\mathrm{RNs}$ em repouso. No entanto, é possível visualizar alta dispersão das notas na Figura 4a. Essa dispersão mais elevada das notas referentes à intensidade do fenômeno doloroso pode ser atribuída a dois possíveis motivos: a sensibilidade da dor de cada neonato é única aos mesmos, e a percepção de cada voluntário com relação ao fenômeno dor também é de caráter exclusivo [6]. Em contraste, os resultados da Figura 4b mostram uma menor dispersão dos valores de cada nota, indicando uma maior concordância entre os voluntários participantes com relação ao estado de repouso no qual o RN se encontrava na hora que cada imagem foi capturada. Esse resultado sugere preliminarmente que é mais 


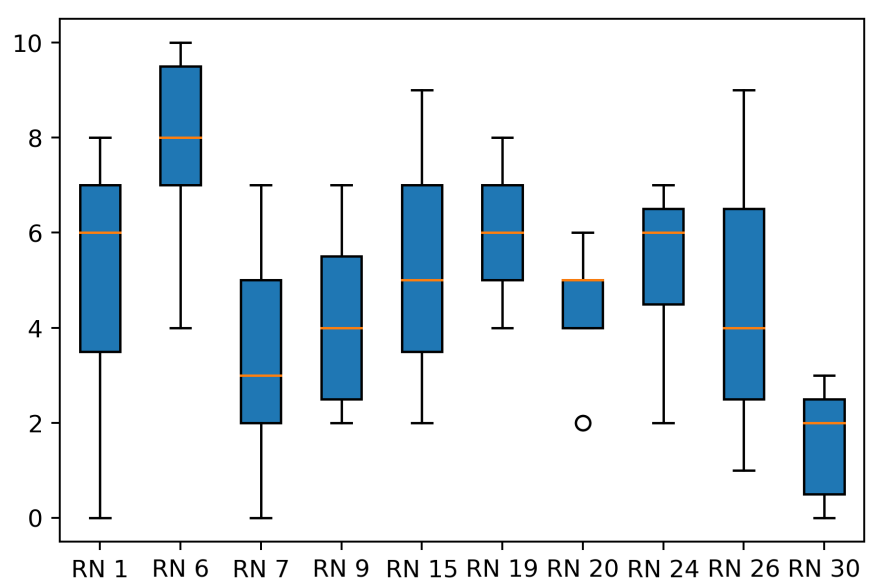

(a) RNs após punção.

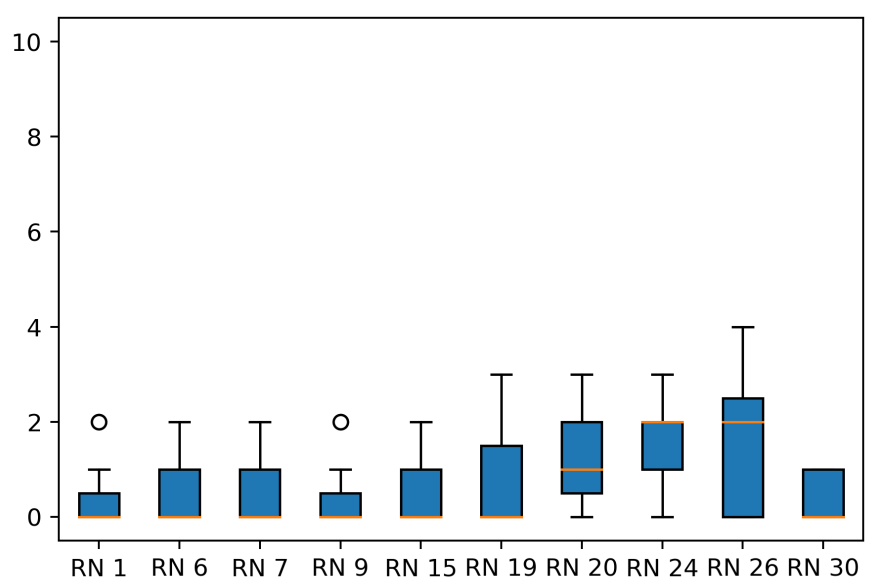

(b) RNs em repouso.

Figura 4: Avaliações do fenômeno dor.

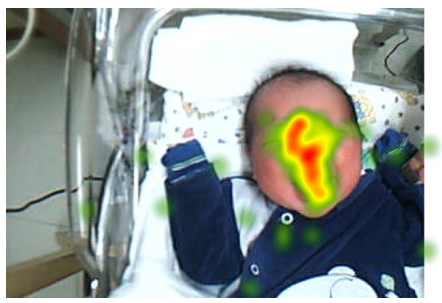

(a) RN 9 após punção.

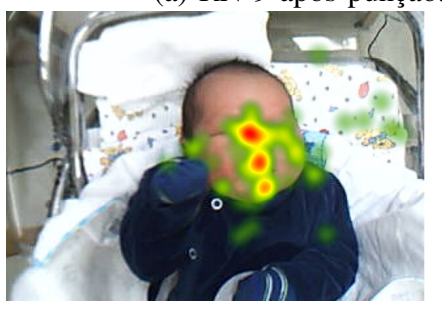

(d) RN 9 em repouso.

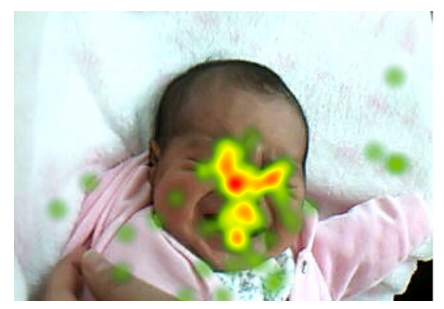

(b) RN 19 após punção.

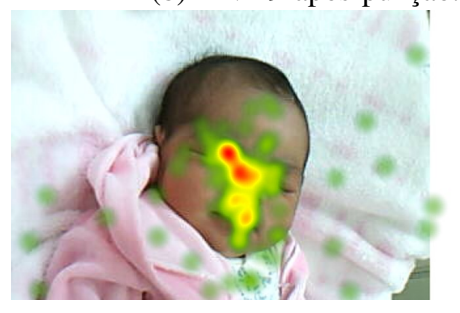

(e) $\mathrm{RN} 19$ em repouso.

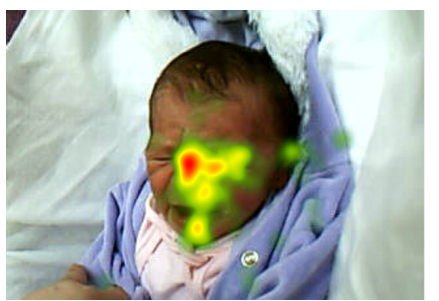

(c) RN 24 após punção.

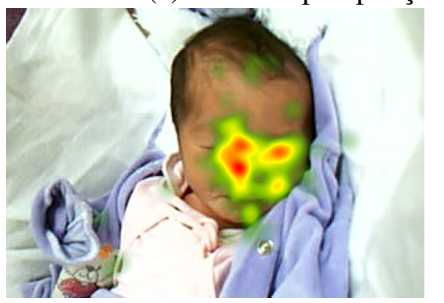

(f) $\mathrm{RN} 24$ em repouso.

Figura 5: Mapas de calor do olhar médio.

fácil julgar a ausência da dor do que julgar a intensidade da mesma nos RNs.

A Figura 5 exibe os dados oculares médios obtidos de todos os participantes para três RNs, após punção e em repouso. A intensidade das cores varia de acordo com a quantidade de fixações em determinado local. Verifica-se um interesse geral pelas regiões dos olhos, nariz e boca dos RNs, demonstrados pelas fortes cores vermelhas do mapa de calor nessas regiões. Também é possível visualizar algumas fixações de olhar em objetos pertencentes ao leito dos RNs. No entanto, como mostrado pelas cores verdes escuras nessas regiões, os voluntários tiveram pouco interesse nesses objetos, confirmando que quando há presença de face humana em determinada cena de estímulo, a mesma tende a atrair a atenção das pessoas, tirando-se o foco de outros objetos pertencentes a cena [16] [17].

De acordo com os mapas de calor exibidos na Figura 6, é possível verificar percepções faciais distintas com relação à avaliação do fenômeno dor entre os participantes. Verifica-se também que os participantes exemplificados em tais imagens tendem a manter suas estratégias oculares durante o experimento, com imagens de $\mathrm{RN}$ em repouso e após punção.

Mais detalhadamente, o participante 1 mostra uma tendência de olhar para as regiões próximas a bochecha esquerda do $\mathrm{RN}$, assim como na fenda palpebral esquerda do mesmo, curiosamente características pertencentes a NFCS, evidenciado pelo forte tom vermelho. Apesar de demonstrar maior concentração na face do RN na imagem após punção, houve uma certa dispersão em outros objetos da imagem do RN em repouso, no entanto, essas fixações mostram-se menos expressivas, exibidas pelos tons verdes nessas regiões.

Em relação ao participante 2, é possível verificar, por meio dos tons vermelho e amarelo, um interesse maior pelas regiões dos olhos, nariz e boca na avaliação do RN após punção, sendo 


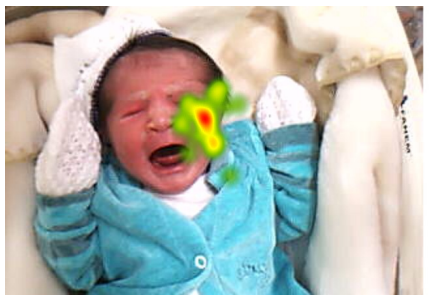

(a) Participante 1 - RN após punção.

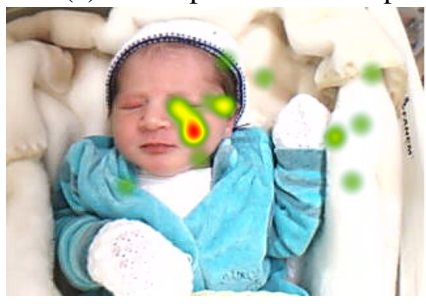

(d) Participante 1 - RN em repouso.

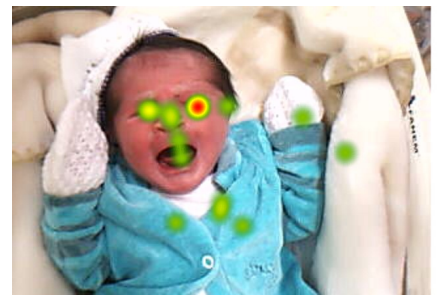

(b) Participante 2 - RN após punção.

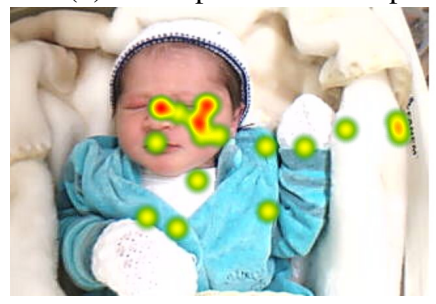

(e) Participante 2 - $\mathrm{RN}$ em repouso.

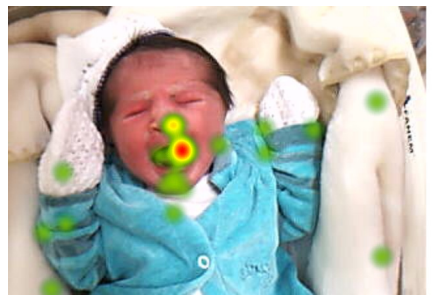

(c) Participante 3 - RN após punção.

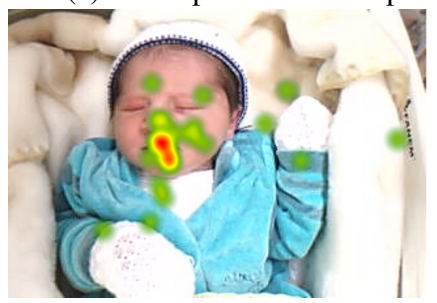

(f) Participante 3 - RN em repouso.

Figura 6: Comparação do olhar entre participantes no RN 15.

também as mesmas regiões de análise da NFCS. No entanto, quando avaliando o $\mathrm{RN}$ em repouso, o participante apresentou maiores dispersões do olhar em outros objetos distintos da face, mostrado pelos tons amarelos mais expressivos.

Por fim, o participante 3 demonstrou um interesse maior pela região da boca e sulco nasolabial, evidenciados pelos tons de vermelho e amarelo, em ambas as imagens do RN. E de maneira semelhante aos participantes exemplificados anteriormente, o participante não demonstrou interesse expressivo em outros objetos da imagem além da própria face.

\section{CONCLUSÃo}

O procedimento descrito neste trabalho apresentou resultados promissores com relação a uma compreensão maior a respeito das características intrínsecas da face do RN necessárias para a avaliação do fenômeno doloroso. A partir dos dados obtidos nessas sessões, foi possível verificar, de forma inédita, estratégias oculares distintas entre os participantes. Dessa forma, foram observados diferentes métodos individuais de análise e percepção facial com relação a dor.

Considerando que os participantes desse estudo não são profissionais da saúde, e, portanto, não possuem treinamento e conhecimento na área, levanta-se a hipótese de que as características intrínsecas das faces de RNs que caracterizam a presença e intensidade do fenômeno doloroso estão presentes nas regiões de análise dos sistemas utilizados por profissionais da saúde [13].

No entanto, após análise dos resultados das avaliações dos RNs por meio da escala analógica proposta, é possível verificar uma maior concordância entre as notas de RNs em repouso. Dessa forma, a análise da presença da dor se apresenta mais fácil do que a análise da intensidade desse mesmo fenômeno, visto a maior dispersão das notas quando as imagens de RNs após punção são avaliadas. Portanto, parece ser mais fácil julgar a ausência da dor do que sua intensidade caso esta esteja presente.
Como trabalhos futuros, pretende-se ampliar a participação de voluntários com profissionais e técnicos da saúde, permitindo uma maior compreensão da percepção facial com relação ao fenômeno dor por parte dos especialistas da área em questão.

\section{AGRADECIMENTOS}

Os autores deste trabalho gostariam de agradecer o apoio da FEI (bolsa PBIC 126/18), da FAPESP (2018/13076-9), e a todos os voluntários que participaram dos experimentos.

\section{REFERÊNCIAS}

[1] G. Zamzmi, R. Kasturi, D. Goldgof, R. Zhi, T. Ashmeade, and Y. Sun, "A review of automated pain assessment in infants: Features, classification tasks, and databases," IEEE reviews in biomedical engineering, vol. 11, pp. 77-96, 2017.

[2] T. M. Heiderich, A. T. F. S. Leslie, and R. Guinsburg, "Neonatal procedural pain can be assessed by computer software that has good sensitivity and specificity to detect facial movements," Acta Paediatrica, vol. 104, no. 2, pp. e63-e69, 2015.

[3] G. F. Teruel, T. M. Heiderich, R. Guinsburg, and C. E. Thomaz, "Analysis and recognition of pain in $2 \mathrm{~d}$ face images of full term and healthy newborns," Proceedings of the XV Encontro Nacional de Inteligencia Artificial, ENIAC 2018, pp. 228-239, 2018.

[4] L. S. Elias, R. Guinsburg, C. A. Peres, R. C. Balda, A. Santos et al., "Disagreement between parents and health professionals regarding pain intensity in critically ill neonates," Jornal de pediatria, vol. 84, no. 1 , pp. 35-40, 2008.

[5] R. d. C. X. Balda, R. Guinsburg, M. F. B. de Almeida, C. de Araújo Peres, M. H. Miyoshi, and B. I. Kopelman, "The recognition of facial expression of pain in full-term newborns by parents and health professionals," Archives of pediatrics \& adolescent medicine, vol. 154, no. 10, pp. 1009-1016, 2000.

[6] H. Merskey, "On the development of pain," Headache: The Journal of Head and Face Pain, vol. 10, no. 3, pp. 116-123, 1970.

[7] IASP, "Iasp publication, pain terms: a list with definitions and notes on usage," Pain, 1979.

[8] L. Diatchenko, A. G. Nackley, G. D. Slade, R. B. Fillingim, and W. Maixner, "Idiopathic pain disorders-pathways of vulnerability," Pain, vol. 123, no. 3, pp. 226-230, 2006.

[9] K. J. Anand, P. R. Hickey et al., "Pain and its effects in the human neonate and fetus," $N$ Engl j Med, vol. 317, no. 21, pp. 1321-1329, 1987. 
[10] J. E. Beyer, D. E. DeGood, L. C. Ashley, and G. A. Russell, "Patterns of postoperative analgesic use with adults and children following cardiac surgery," Pain, vol. 17, no. 1, pp. 71-81, 1983.

[11] K. J. Anand and D. B. Carr, "The neuroanatomy, neurophysiology, and neurochemistry of pain, stress, and analgesia in newborns and children," Pediatric Clinics of North America, vol. 36, no. 4, pp. 795-822, 1989.

[12] B. Golianu, E. J. Krane, K. S. Galloway, and M. Yaster, "Pediatric acute pain management," Pediatric Clinics of North America, vol. 47, no. 3, pp. 559-587, 2000.

[13] R. V. Grunau and K. D. Craig, "Pain expression in neonates: facial action and cry," Pain, vol. 28, no. 3, pp. 395-410, 1987.

[14] R. V. Grunau, C. C. Johnston, and K. D. Craig, "Neonatal facial and cry responses to invasive and non-invasive procedures," Pain, vol. 42, no. 3, pp. 295-305, 1990.

[15] K. Holmqvist, M. Nyström, R. Andersson, R. Dewhurst, H. Jarodzka, and J. Van de Weijer, Eye tracking: A comprehensive guide to methods and measures. OUP Oxford, 2011.

[16] V. Bruce and A. Young, Face Perception. East Sussex, England: Taylor \& Francis, 2013.

[17] O. Hershler and S. Hochstein, "At first sight: A high-level pop out effect for faces," Vision research, vol. 45, no. 13, pp. 1707-1724, 2005. 